\title{
Use of video-based multimedia information to reduce anxiety before office hysteroscopy
}

\author{
Aysu Akca ${ }^{1}$, Gulseren Yilmaz ${ }^{2}$, Aytul Corbacioglu Esmer ${ }^{1}$, Semra Yuksel ${ }^{3}$, Nadiye Koroglu ${ }^{1}$, Berna Aslan Cetin ${ }^{1}$ \\ ${ }^{1}$ Department of Obstetrics and Gynecology, University of Health Sciences, Faculty of Medicine, Kanuni Sultan Suleyman Hospital, \\ Istanbul, Turkey \\ ${ }^{2}$ Department of Anesthesiology and Reanimation, University of Health Sciences, Faculty of Medicine, Kanuni Sultan Suleyman \\ Hospital, Istanbul, Turkey \\ ${ }^{3}$ Department of Obstetrics and Gynecology, University of Health Sciences, Faculty of Medicine, GOP Taksim Hospital, Istanbul, Turkey
}

Videosurgery Miniinv 2020; 15 (2): 329-336

DOI: https://doi.org/10.5114/wiitm.2019.89378

\begin{abstract}
Introduction: Use of multimedia tools has been shown to improve patient comprehension, reduce pre-procedural anxiety, and increase patient satisfaction in various surgical settings.

Aim: To investigate the impact of video-based multimedia information (MMI) on the anxiety levels of patients undergoing office hysteroscopy $(\mathrm{OH})$.

Material and methods: All consecutive women aged 18-65 years and scheduled for diagnostic $\mathrm{OH}$ were enrolled in this prospective randomized study. Subjects were assigned to receive video-based $\mathrm{MMI}$ or conventional written information (controls). The trait and state anxiety were assessed using the State and Trait Anxiety Inventory (STAI) before the MMI or written information. STAI-state (STAI-S) was repeated after application of the MMI or written information. All patients underwent a standardized transvaginal hysteroscopy procedure by the same gynecologist. Following the hysteroscopy, patient satisfaction and procedural pain were ranked using a Likert scale and visual analogue scale.

Results: Fifty-two patients were randomized to receive a video-based $\mathrm{MMI}$, and 52 patients were randomized to receive written information. Post-information STAI-S score was significantly lower in the MMI group than that of the written information group (45.0 \pm 8.0 vs. $49.4 \pm 8.4, p<0.001,95 \% \mathrm{Cl}$ for the difference: $1.36-7.79)$. Moreover, the satisfaction rate of the video group was significantly higher than the satisfaction rate of the controls $(92.3 \% \mathrm{vs}$. $63.5 \%, p<0.001)$. VAS score of procedural pain was similar for the two groups.

Conclusions: $A$ video-based $\mathrm{MMI}$ before $\mathrm{OH}$ might be preferred to conventional information methods in order to reduce the pre-procedural anxiety and to increase patients' satisfaction.
\end{abstract}

Key words: anxiety, office hysteroscopy, satisfaction, pain.

\section{Introduction}

Hysteroscopy is a well-recognized technique for the investigation of thickened endometrial lining on ultrasonography, suspected Mullerian anomalies, and abnormal uterine bleeding. Evidence concerning the safety and the success of hysteroscopy in the hospital setting led to the employment of this technique also in the outpatient setting with both diagnostic and therapeutic potential, and increasing patient compliance [1]. Although hysteroscopy is described as a minimally invasive technique, the term 'minimally invasive' does not necessarily take into

\section{Address for correspondence}

Aysu Akca, Department of Obstetrics and Gynecology, University of Health Sciences, Faculty of Medicine, Kanuni Sultan Suleyman

Hospital, Istanbul, Turkey, e-mail: aysuakca4@gmail.com 
account patients' emotional experience. A high level of anxiety measured with the Spielberger State-Trait Anxiety Index (STAI) has been observed in patients undergoing office hysteroscopy $(\mathrm{OH})$ [2].

Anxiety refers to an abnormal and overwhelming sense of apprehension and fear, often marked by physiological signs [3]. The most recognized and studied manifestation of patient anxiety in association with a medical encounter is the white coat effect [4]. Anxiety related to surgery is also a serious problem associated with negative consequences before and after the surgery, including elevation in blood pressure and dysrhythmia [5]. Despite the less invasive nature of the office hysteroscopy compared to many surgical procedures, pre-procedural anxiety is still a critical issue affecting patients' satisfaction $[6,7]$. A previous study on women undergoing $\mathrm{OH}$ revealed that $80 \%$ of the women had a moderate to severe anxiety state, defined by the authors as a state anxiety value $\geq 34$ [8].

Pharmacologic interventions, including anxiolytics and sedatives, have been used to overcome the preoperative anxiety in the inpatient setting. However, due to the short duration of the $\mathrm{OH}$ and the potential side effects of the agents used for anxiolysis, the implementation of non-pharmacological tools would be preferable, in line with the minimalistic nature of the $\mathrm{OH}$. Use of multimedia tools as an adjunct to conventional consent has been shown to improve patient comprehension, reduce pre-procedural anxiety, and increase patient satisfaction in various surgical settings [9]. However, data concerning the role of video-based multimedia information (MMI) before the $\mathrm{OH}$ have not been studied yet.

\section{Aim}

In this context, we aimed to investigate the impact of video-based MMI on anxiety and satisfaction of patients undergoing $\mathrm{OH}$.

\section{Material and methods}

\section{Patient selection}

All women aged between 18 and 65 years and scheduled for diagnostic office hysteroscopy between April 2019 and July 2019 were enrolled in this randomized, prospective study. Women with an established indication for diagnostic hysteroscopy including abnormal premenopausal or postmenopausal uterine bleeding, the need for removal of an intrauterine device, suspected Mullerian anomalies and confirmation of abnormal test findings such as abnormal hysterosalpingography (HSG) or thickened endometrial lining on ultrasonography were recruited. Exclusion criteria were as follows: patients who require endometrial biopsy, polypectomy, and cervical dilatation with Hegar dilators, the existence of any contraindication for hysteroscopy and the presence of a disease or disability that can intervene in attaining the objective of the study (visual deficit, auditory or sensory severe, illnesses or mental syndromes). Written informed consent was obtained from all subjects included in the study. The study was approved by the Institutional Ethical Committee and was performed in accordance with the recent version of the Helsinki Declaration (2019/92). Additionally, the study was registered with anzctrorg. au (no:2619000645156p). The power calculation was based on our pilot study with the first 18 patients. We used the t-test assessing the difference between two independent means for comparing post-information STAl state (STAI-S) measurements in the two groups (video group: $47.3 \pm 7.5$, STAI-S score of the controls: $42.3 \pm 6.4$, $\alpha$ error: 0.05 , power: 0.95 , effects size: 0.72 ) [10]. The results showed that at least 102 patients (51 patients for each group) were required for an adequate sample size.

\section{State and Trait Anxiety Inventory}

Before randomization, all patients were asked to fill out the STAI, which is a validated and widely used self-report questionnaire assessing both state and trait anxiety [11, 12]. The STAI consists of two parts with 20 questions in each: STAI-S, which intends to evaluate the current state of anxiety, and trait anxiety (STAI-T) that measures long term anxiety levels, and each answer were scored on a scale of 1-4. Responses for the STAI-S scale evaluate the intensity of current feelings "right now": 1) not at all, 2) somewhat, 3) moderately so, and 4) very much so (Figure 1). Responses for the STAI-T scale evaluate the frequency of feelings "in general": 1) almost never, 2) sometimes, 3) often, and 4) almost always (Figure 2). Item scores are added to obtain subtest total scores. The overall score is $20-80$.

\section{Randomization}

Using random allocation software (www.randomization.com), 106 patients who were eligible 
1. I feel calm...

2. I feel secure..

3. I am tense

4. I feel strained

5. I feel at ease

6. I feel upset.

7. I am presently worrying over possible misfortunes

8. I feel satisfied

9. I feel frightened

10. I feel comfortable.

11. I feel self-confident.

12. I feel nervous.

13. I am jittery ....

14. I feel indecisive.

15. I am relaxed...

16. I feel content

17. I am worried

18. I feel confused...

19. I feel steady.....

20. I feel pleasant

Scoring:
1) Not at all
2) Somewhat
3) Moderately so
4) Very much so

Figure 1. State anxiety inventory items

21. I feel pleasant

22. I feel nervous and restless

23. I feel satisfied with myself

24. I wish I could be as happy as others seem to be

25. I feel like a failure

26. I feel rested.

27. I am "calm, cool, and collected"

28. I feel that difficulties are piling up so that I cannot overcome them

29. I worry too much over something that really doesn't matter

30. I am happy...

31. I have disturbing thoughts

32. I lack self-confidence.

33. I feel secure

34. I make decisions easily.

35. I feel inadequate.

36. I am content

37. Some unimportant thought runs through my mind and bothers me.

38. I take disappointments so keenly that I can't put them out of my mind

39. I am a steady person...

40. I get in a state of tension or turmoil as I think over my recent concerns and interests

$\begin{array}{llll}1 & 2 & 3 & 4 \\ 1 & 2 & 3 & 4 \\ 1 & 2 & 3 & 4 \\ 1 & 2 & 3 & 4 \\ 1 & 2 & 3 & 4 \\ 1 & 2 & 3 & 4 \\ 1 & 2 & 3 & 4 \\ 1 & 2 & 3 & 4 \\ 1 & 2 & 3 & 4 \\ 1 & 2 & 3 & 4 \\ 1 & 2 & 3 & 4 \\ 1 & 2 & 3 & 4 \\ 1 & 2 & 3 & 4 \\ 1 & 2 & 3 & 4 \\ 1 & 2 & 3 & 4 \\ 1 & 2 & 3 & 4 \\ 1 & 2 & 3 & 4 \\ 1 & 2 & 3 & 4 \\ 1 & 2 & 3 & 4 \\ 1 & 2 & 3 & 4\end{array}$

Scoring:

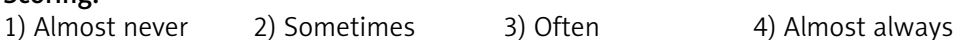

Figure 2. Trait anxiety inventory items

for the study were randomly assigned to one of the study groups. The video-based MMI group included the patients who received an MMI video which explains all details of the procedure (Table I). The control group included patients who received brief written information regarding the procedure before the surgery. Ten minutes after the MMI or written information, the STAI-S questionnaire was repeated in all patients. Following the hysteroscopy, patient satisfaction was evaluated with a four-point likert scale, and procedural pain was ranked using a tenpoint visual analogue scale (VAS) (Figures 3 and 4).

Table I. Subjects operated in the video-based multimedia information group

\begin{tabular}{|l|}
\hline Indications of hysteroscopy \\
\hline Hysteroscopy room \\
\hline Hysteroscopy device \\
\hline Aim of the diagnostic hysteroscopy \\
\hline Steps of the procedure \\
\hline Risks and complications \\
\hline Length of the procedure and hospital stay \\
\hline Post-procedure recommendations \\
\hline
\end{tabular}




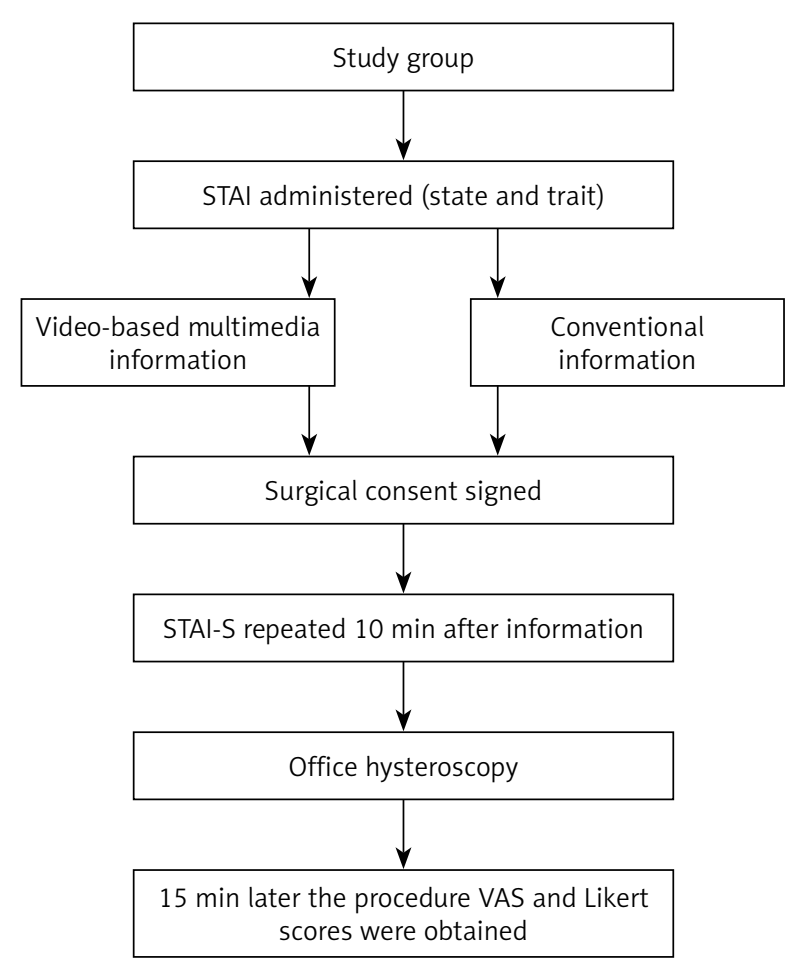

Figure 3. Study protocol

\section{Hysteroscopy}

All patients underwent a standardized transvaginal hysteroscopy procedure by the same gynecologist who had professional experience of $>8$ years in hysteroscopy and has been certified to perform hysteroscopy by an independent committee. A $5 \mathrm{~mm}$ Bettocchi hysteroscope (Karl Storz $\mathrm{GmbH} \&$ Co, Tuttlingen, Germany) was used with physiological serum distension medium and initial irrigation pressure of $75 \mathrm{~mm} \mathrm{Hg}$. Following the completion of the hysteroscopy and $15 \mathrm{~min}$ after the patient had got dressed; the pain was assessed using the VAS (0; no pain, 10; most severe pain imaginable). Before discharge, a four-point Likert scale to determine the patients' satisfaction (0; not satisfied, 1; poorly satisfied, 2; satisfied, 3; very satisfied) was administered. A Likert scale score $\geq$ two was assumed as satisfaction.

\section{Primary and secondary outcome}

The aim of this study was to investigate the impact of $\mathrm{MMI}$ on the anxiety levels in patients undergoing $\mathrm{OH}$. The difference in the procedural satisfaction rate between the groups was the secondary outcome measure.

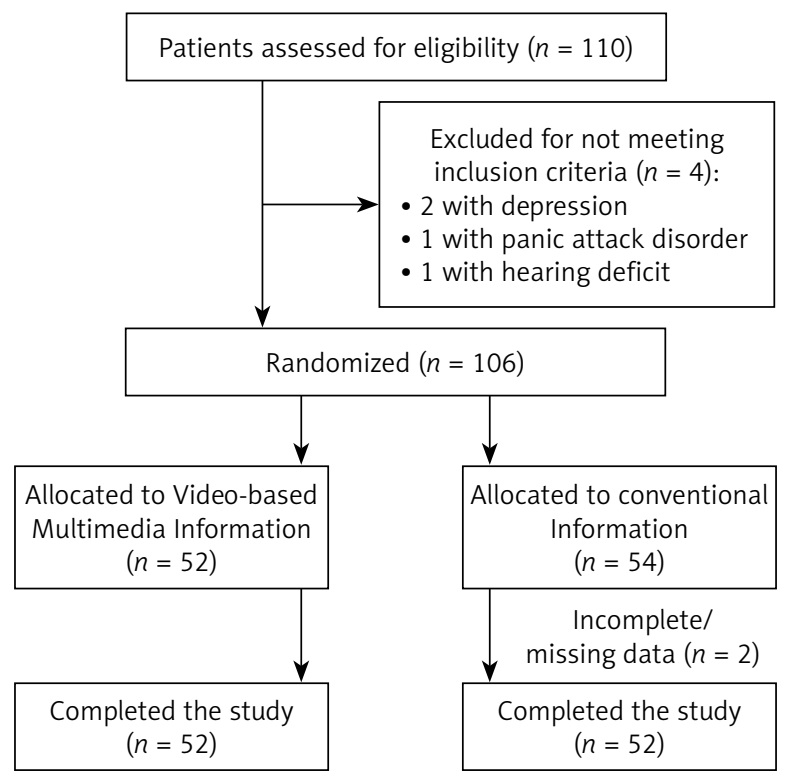

Figure 4. Flowchart demonstrating subject enrollment

\section{Statistical analysis}

Statistical analyses were carried out using SPSS for Windows, version 17 (SPSS, Chicago, IL, USA). Continuous variables were presented as mean \pm standard deviation (mean \pm SD) and categorical variables as frequency $(n)$ and percentage (\%). The comparison of the two groups was performed with Student's $t$-test, Mann-Whitney $U$-test, $\chi^{2}$ test or Fisher's exact test, where appropriate. The paired samples $t$-test was used for the comparison of preand post- informational STAI-S scores of the two groups. Two-sided $p$-value $\leq 0.05$ was interpreted as statistically significant.

\section{Results}

A total of 104 patients (mean age: $38.8 \pm 8.6$ years) were enrolled in the study. Fifty-two patients were randomized to the video group, and 52 were randomized to the control group. The two groups were similar with regard to age, parity, body mass index, menopausal and educational status, STAI-T score, and the hysteroscopy indications (Table II). The pre-informational STAI-S was also similar for the two groups. Post-informational STAI-S score was significantly lower than the pre-informational STAI-S score in the video group $(49.0 \pm 8.0$ vs. $45.0 \pm 8.0, p>0.001,95 \% \mathrm{Cl}$ for the difference: $2.60-5.28$ ), whereas no significant change occurred in STAI-S score in the control group 
Table II. Demographic and clinical features of the study population

\begin{tabular}{|lccc|}
\hline Parameter & $\begin{array}{c}\text { Video-based MMl group } \\
(n=52)\end{array}$ & $\begin{array}{c}\text { Control group } \\
(n=52)\end{array}$ & P-value \\
\hline Age [years] & $39.5 \pm 7.4$ & $38.3 \pm 9.2$ & 0.28 \\
\hline Parity $(n)$ & $2.0 \pm 1.3$ & $1.8 \pm 1.2$ & 0.52 \\
\hline Nulliparity $(n)$ & $9(17.3 \%)$ & $7(13.5 \%)$ & 0.78 \\
\hline BMI $\left[\mathrm{kg} / \mathrm{m}^{2}\right]$ & $26.7 \pm 5.3$ & $26.6 \pm 4.2$ & 0.65 \\
\hline Postmenopausal patient $(n)$ & $6(11.5 \%)$ & $5(9.6 \%)$ & 0.92 \\
\hline Graduated from at least high school $(n)$ & $37(71.2 \%)$ & $33(63.5 \%)$ & 0.53 \\
\hline \begin{tabular}{l} 
Indications $(n):$ \\
\hline AUB
\end{tabular} & $40(76.9 \%)$ & $39(75 \%)$ & 0.82 \\
\hline PMB & $4(7.6 \%)$ & $5(9.6 \%)$ & $7(13.4 \%)$ \\
\hline Infertility & $6(11.5 \%)$ & $1(1.9 \%)$ & 0.60 \\
\hline Secondary amenorrhea & $2(3.8 \%)$ & $44.2 \pm 7.2$ & \\
\hline STAI-T & $43.8 \pm 9.7$ & & \\
\hline
\end{tabular}

Data are given as mean $\pm S D$ or frequency and percentage. AUB - abnormal uterine bleeding, PMB - postmenopausal bleeding, STAI-T - State-Trait Anxiety Index-Trait, MMI-multi-media information.

Table III. Comparison of STAI-state score before and after the video-based MMI or written information in the two groups

\begin{tabular}{|lccc|}
\hline Parameter & $\begin{array}{c}\text { Video-based MMI group } \\
(n=52)\end{array}$ & $\begin{array}{c}\text { Control group } \\
(n=52)\end{array}$ & $P$-value \\
\hline STAI-S pre-informational & $49.0 \pm 8.0$ & $49.3 \pm 8.3$ & 0.49 \\
\hline STAI-S post-informational & $45.0 \pm 8.0$ & $49.4 \pm 8.4$ & $<0.001$ \\
\hline -value' & $<0.001$ & 0.15 & \\
\hline
\end{tabular}

Data are given as mean \pm SD. STAI-S - State-Trait Anxiety Index-State, MMI- multi-media information. P-value - p-value derived from the comparison of the video and control groups. P-value' - $p$-value derived from the comparison of the pre-and post-informational STAI-S scores.

$(49.3 \pm 8.3$ vs. $49.4 \pm 8.4, p=0.15,95 \% \mathrm{Cl}$ for the difference: $-0.18-0.03$, Table III, Figure 5). Moreover, the satisfaction rate of the patients receiving video information before the hysteroscopy was significantly higher than the satisfaction rate of the controls (92.3\% vs. $63.5 \%, p<0.001)$ and the VAS score of procedural pain was similar for the two groups (Table IV).

\section{Discussion}

The present study demonstrates that not conventional information, but $\mathrm{MMI}$ provides a significant reduction in pre-procedural anxiety in patients undergoing $\mathrm{OH}$. Our findings also indicate that patients' satisfaction is significantly higher in the group receiving $\mathrm{MMI}$ than that of the conventional information group.
In the last few decades, the concept of inpatient care of patients for several minor interventions has shifted towards outpatient delivery of health care, across all medical specialties [13]. The outpatient setting, for the majority of minor interventions, brought several advantages for both patients and the health-care providers by means of the comfortable setting and the reduction in the medical costs [14]. However, the advantages obtained with the outpatient delivery of health care might be offset by increased anxiety and related dissatisfaction. Available data on the level of anxiety before $\mathrm{OH}$ have demonstrated that the anxiety experienced before hysteroscopy is at least comparable to that experienced by women undergoing gynecological surgery with general anesthesia [15]. In a study by Gupta 


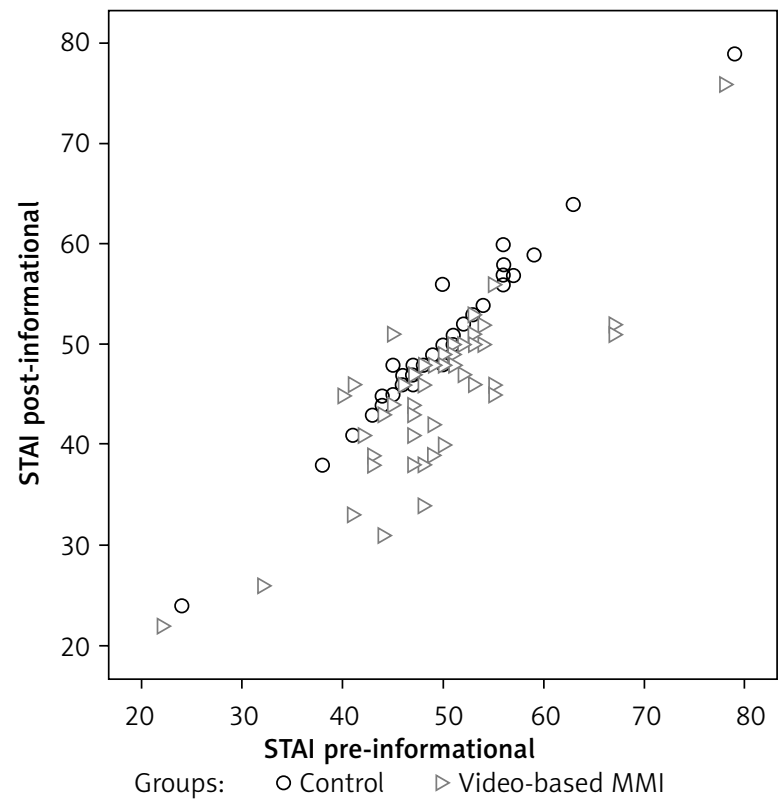

Figure 5. Pre- and post-information STAI-state scores of the patients receiving video-based $\mathrm{MMI}$ or written information

et al., one-fifth of the women undergoing $\mathrm{OH}$ were shown to have severe anxiety, which is greater than that found among women awaiting major surgery [6]. The increased anxiety in outpatients was attributed to the perception of the patient that extensive interventional procedures could be carried out in the outpatient setting. Being middle-aged, infertility, suspected malignity as the indication for hysteroscopy, waiting $60 \mathrm{~min}$ or more for the procedure, and operative hysteroscopy rather than diagnostic hysteroscopy have been found to cause anxiety in women undergoing $\mathrm{OH}$ [15-19].

Although $\mathrm{OH}$ is well tolerated by the majority of the women, it can still be a painful experience for some. Insertion of the speculum, cervical manipulation, insertion of the hysteroscope, and the uterine distension may cause pain during the $\mathrm{OH}$ [20]. Several methods of pain relief have been described for $\mathrm{OH}$. These include paracetamol, non-steroidal anti-inflammatory drugs, opioids, and local anesthesia [21]. Paracervical block, intracervical anesthesia, intrauterine anesthesia, and topical anesthesia with sprays have been used for this purpose [22, 23]. However, the results are inconclusive, and there is no consensus on the use of analgesia [24, 25].

Anxiety has been shown to act as a risk factor for the experience of significant pain [26]. Influential preoperative communication and patient education are effective tools for reducing preoperative anxiety. The provision of appropriate information regarding the procedure for which they are scheduled and addressing their concerns may reduce the anxiety and worry about the forthcoming procedure. Answering patients' questions concerning diagnosis and treatment with straightforwardness has been reported to reduce preoperative anxiety and increase patients' satisfaction [27]. Clarity has been shown to be superior to the amount of the information given for reducing the pre-procedural anxiety in patients undergoing gastrointestinal endoscopy [28]. However, the establishment of a faithful and confidential physician-patient relationship frequently requires a longer time than expected. The troubles in creating effective communication with the patient and providing satisfying answers to the questions in their mind could be defeated by the implementation of multimedia approaches, which appears to increase patients' understanding of the procedure and improve satisfaction. A systematic review which included 33 studies reported that the adoption of MMI with video clips, Microsoft power-point presentations or interactive programs as an adjunct to conventional written information was associated with improved patients' comprehension in subjects undergoing a variety of surgical or interventional procedures [9]. However, a significant reduction in pre-procedural anxiety with the MMI was demonstrated only in a few studies. In a previous study conducted by Stergiopoulou et al., a significant reduction in patients' anxiety with an interactive MME program was reported in patients

Table IV. Procedural satisfaction and pain scores of the study groups

\begin{tabular}{|lccc|}
\hline Variable & $\begin{array}{c}\text { Video-based MMI group } \\
(n=52)\end{array}$ & $\begin{array}{c}\text { Control group } \\
(n=52)\end{array}$ & $P$-value \\
\hline VAS score & $4.5 \pm 2.0$ & $4.9 \pm 2.5$ & 0.39 \\
\hline Satisfied with procedure $(n)$ & $48(92.3 \%)$ & $33(63.5 \%)$ & $<0.001$ \\
\hline
\end{tabular}

Data are given as mean $\pm S D$ or frequency and percentage. VAS - visual analogue scale, $M M I-$ multi-media information. 
undergoing laparoscopic cholecystectomy [29]. Luck and colleagues in their study with patients scheduled to undergo colonoscopy found that patients who received video-based $\mathrm{MMI}$ were significantly less anxious before colonoscopy than those who did not [30]. The efficacy of MMI was also studied in the neighboring field of colposcopy. Freeman-Wang et al. found in patients undergoing colposcopy that the introduction of visual information in the form of an explanatory video prior to the procedure significantly reduced anxiety [31].

Our findings, for the first time, have demonstrated that the use of video-based MMI leads to a significant reduction in state anxiety in patients undergoing $\mathrm{OH}$. Our findings support the previous evidence obtained in colonoscopy, colposcopy, and laparoscopic cholecystectomy with MMI. Our results also show that self-reported procedural satisfaction is significantly higher in patients who receive $\mathrm{MMI}$ prior to $\mathrm{OH}$ than those who do not. We speculate that MMI may improve the recovery process besides reducing the anxiety. The association of detailed patient information with faster recovery, which was reported by Wallace, supports this consideration [32]. Nevertheless, the MMI failed to provide a reduction in procedural pain. Many distinct factors, such as the presence of cervical stenosis or previous undesirable experiences with office interventions, might contribute to the pain perception. Existence of these factors might have influenced the pain perception in the subjects of the present study.

Several limitations of this study should be considered, including the lack of participation of illiterate patients who might have benefited the most from multimedia education, and the lack of a comparison of hemodynamic data. Also, only patients who underwent hysteroscopy for diagnostic purposes were enrolled in this study. The impact of the MMI in patients undergoing $\mathrm{OH}$ for several interventions should be addressed in further studies. In addition, we did not use novel hormonal therapy agents, such as dienogest, which has been shown to improve endometrial thinning $[33,34]$. Implementation of such an agent could facilitate the hysteroscopy by offering a clearer view of the uterine cavity and therefore influence the patients' anxiety and the complication rate. Elimination of the limitations mentioned above would strengthen the evidence concerning the benefit of $\mathrm{MMI}$ in $\mathrm{OH}$.

\section{Conclusions}

A significant level of anxiety is observed in patients awaiting $\mathrm{OH}$. Our findings demonstrate that video-based $\mathrm{MMI}$ describing the details of the procedure leads to a significant reduction in patients' anxiety levels. Moreover, patients' satisfaction is significantly higher in those receiving $\mathrm{MMI}$ prior to $\mathrm{OH}$ compared to patients receiving only written information. Given the superiority of MMI over conventional written information, visual information in the form of an explanatory video prior to $\mathrm{OH}$ might be preferable to conventional information methods.

\section{Conflict of interest}

The authors declare no conflict of interest.

\section{References}

1. Kremer C, Barik S, Duffy S. Flexible outpatient hysteroscopy without anaesthesia: a safe, successful and well tolerated procedure. Br J Obstet Gynaecol 1998; 105: 672-6.

2. Gambadauro P, Navaratnarajah R, Carli V. Anxiety at outpatient hysteroscopy. Gynecol Surg 2015; 12: 189-96.

3. Dean E. Anxiety. Nurs Stand 2016; 30: 15.

4. Ogedegbe G, Pickering TG, Clemow L, et al. The misdiagnosis of hypertension: the role of patient anxiety. Arch Intern Med 2008; 168: 2459-65.

5. Vetter D, Barth J, Uyulmaz S et al. Effects of art on surgical patients: a systematic review and meta-analysis. Ann Surg 2015; 262: 704-13

6. Gupta J, Clark T, More S, Pattison H. Patient anxiety and experiences associated with an outpatient "one-stop" "see and treat” hysteroscopy clinic. Surg Endosc 2004; 18: 1099-104.

7. Kokanali MK, Cavkaytar S, Guzel Al, et al. Impact of preprocedural anxiety levels on pain perception in patients undergoing office hysteroscopy. J Chin Med Assoc 2014; 77: 477-81.

8. Carta G, Palermo P, Marinangeli F, et al. Waiting time and pain during office hysteroscopy. J Minim Invasive Gynecol 2012; 19: 360-4.

9. Nehme J, El-Khani U, Chow A, et al. The use of multimedia consent programs for surgical procedures: a systematic review. Surg Innov 2013; 20: 13-23.

10. Faul F, Erdfelder E, Buchner A, Lang AG. Statistical power analyses using $G^{*}$ Power 3.1: tests for correlation and regression analyses. Behav Res Methods 2009; 41: 1149-60.

11. Guillen-Riquelme A, Buela-Casal G. [Meta-analysis of group comparison and meta-analysis of reliability generalization of the State-Trait Anxiety Inventory Questionnaire (STAI)]. Rev Esp Salud Publica 2014; 88: 101-12.

12. Julian LJ. Measures of anxiety: State-Trait Anxiety Inventory (STAI), Beck Anxiety Inventory (BAI), and Hospital Anxiety and Depression Scale-Anxiety (HADS-A). Arthritis Care Res (Hoboken) 2011; 63 Suppl 11: S467-72. 
13. Sulkes DJ, Scott IU, Flynn HW Jr, Feuer WJ. Evaluating outpatient versus inpatient costs in endophthalmitis management. Retina 2002; 22: 747-51.

14. Clark TJ, Gupta JK. Outpatient hysteroscopy. Obstet Gynaecol 2002; 4: 217-21.

15. Bromberger JT, Kravitz HM, Chang Y, et al. Does risk for anxiety increase during the menopausal transition? Study of Women's Health Across the Nation (SWAN). Menopause 2013; 20: 488-95.

16. Angioli R, Nardone CDC, Plotti F, et al. Use of music to reduce anxiety during office hysteroscopy: prospective randomized trial. J Minim Invasive Gynecol 2014; 21: 454-9.

17. Caprilli S, Baiocco F, Zanetti H, Medina M. Psychological reactions at the outpatient hysteroscopy. Medicina Psicosomatica Roma 2000; 45: 11-22.

18. Carta G, Palermo P, Marinangeli F, et al. Waiting time and pain during office hysteroscopy. J Minim Invasive Gynecol 2012; 19: 360-4.

19. Heyer CM, Thüring J, Lemburg SP, et al. Anxiety of patients undergoing CT imaging - an underestimated problem? Acad Radiol 2015; 22: 105-12.

20. Lau WC, Lo WK, Tam WH, Yuen PM. Paracervical anaesthesia in outpatient hysteroscopy: a randomised double-blind placebo-controlled trial. Br J Obstet Gynaecol 1999; 106: 356-9.

21. Yang J, Vollenhoven B. Pain control in outpatient hysteroscopy. Obstet Gynecol Surv 2002; 57: 693-702.

22. Cooper NA, Khan KS, Clark TJ. Local anaesthesia for pain control during outpatient hysteroscopy: systematic review and metaanalysis. BMJ 2010; 340: c1130.

23. Mercier RJ, Zerden ML. Intrauterine anesthesia for gynecologic procedures: a systematic review. Obstet Gynecol 2012; 120: 669-77.

24. O’Flynn H, Murphy LL, Ahmad G, Watson AJ. Pain relief in outpatient hysteroscopy: a survey of current UK clinical practice. Eur J Obstet Gynecol Reprod Biol 2011; 154: 9-15.

25. Amer-Cuenca JJ, Marin-Buck A, Vitale SG, et al. Non-pharmacological pain control in outpatient hysteroscopies. Minim Invasive Ther Allied Technol 2019; doi: 10.1080/13645706.2019.1576054.

26. De laco P, Marabini A, Stefanetti M, et al. Acceptability and pain of outpatient hysteroscopy. J Am Assoc Gynecol Laparosc 2000; 7: 71-5.

27. Lim L, Chow P, Wong CY, et al. Doctor-patient communication, knowledge, and question prompt lists in reducing preoperative anxiety: a randomized control study. Asian I Surg 2011; 34: 175-80.

28. Eberhardt J, Van Wersch A, Van Schaik P, Cann P. Information, social support and anxiety before gastrointestinal endoscopy. Br J Health Psychol 2006; 11: 551-9.

29. Stergiopoulou A, Birbas K, Katostaras T, Mantas J. The effect of interactive multimedia on preoperative knowledge and postoperative recovery of patients undergoing laparoscopic cholecystectomy. Methods Inf Med 2007; 46: 406-9.

30. Luck A, Pearson S, Maddern G, Hewett P. Effects of video information on precolonoscopy anxiety and knowledge: a randomised trial. Lancet 1999; 354: 2032-5.
31. Freeman-Wang T, Walker P, Linehan J, et al. Anxiety levels in women attending colposcopy clinics for treatment for cervical intraepithelial neoplasia: a randomised trial of written and video information. BJOG 2001; 108: 482-4.

32. Wallace LM. Communication variables in the design of pre-surgical preparatory information. Br J Clin Psychol 1986; 25: 111-8.

33. Kodama M, Onoue M, Otsuka H, et al. Efficacy of dienogest in thinning the endometrium before hysteroscopic surgery. J Minim Invasive Gynecol 2013; 20: 790-5.

34. Lagana AS, Vitale SG, Muscia V, et al. Endometrial preparation with Dienogest before hysteroscopic surgery: a systematic review. Arch Gynecol Obstet 2017; 295: 661-7.

Received: 18.08.2019, accepted: 3.10.2019. 